\title{
Supplementary Information for Flexible Solid-State Electrolyte with Aligned Nanostructures Derived from Wood
}

Jiaqi Dai1, a, Kun (Kelvin) Fu1, 2, a, Yunhui Gong, 2, a, Jianwei Song1, Chaoji Chen'1, Yonggang Yao $^{1}$, Glenn Pastel ${ }^{1}$, Lei Zhang ${ }^{2}$, Eric Wachsman ${ }^{1,2, *}$, Liangbing $\mathrm{Hu}^{1,2, *}$

${ }^{1}$ Department of Materials Science and Engineering, University of Maryland, College Park, MD 20742;

${ }^{2}$ Maryland Energy Innovation Institute, University of Maryland, College Park, MD 20742.

a These authors contributed equally.

*To whom correspondence may be addressed. Email: binghu@umd.edu or ewach@umd.edu 
Figure S1. Photograph of pristine wood. A piece of basswood, whose cross-section shows a naturally aligned structure. 


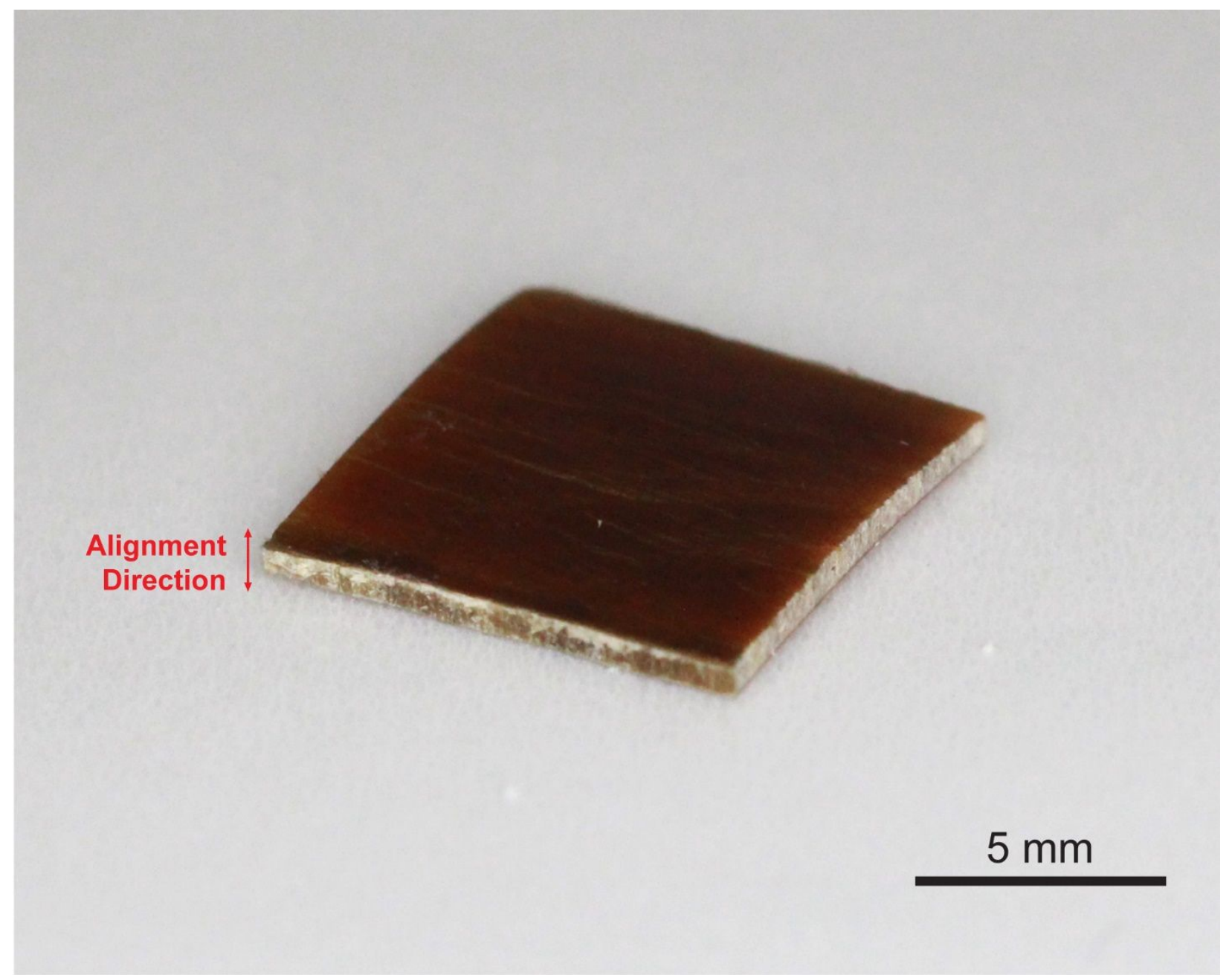

Figure S2. Photograph of compressed wood (wood template). The wood template fabricated from basswood through partial lignin removal and mechanical compression. The wood was compressed perpendicular to its longitudinal direction (natural growth direction), which effectively densifies the wood template while maintaining the natural aligned structure. 

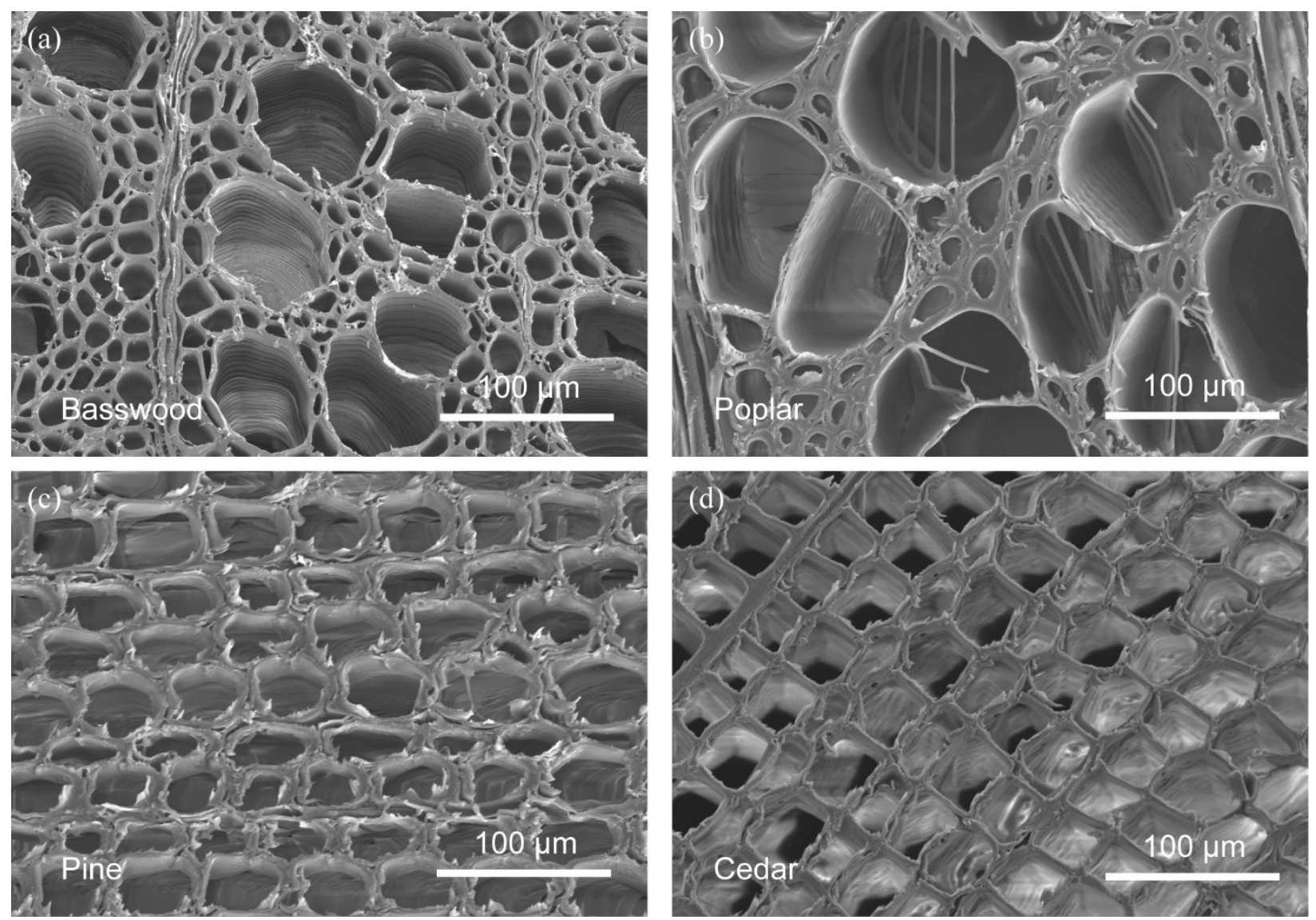

Figure S3. Cross-sectional SEM image of (a) hardwood basswood, (b) hardwood poplar, (c) softwood pine, and (d) softwood cedar shows the microstructure differences. Hardwood usually has distributed vessel elements that have large diameters. The pores in hardwood vary a lot in size. Softwood consists mainly of thin-walled tubular tracheid cells, where large areas of similar sized, evenly distributed pores (lumen) can be easily found. Softwood can be employed as a template for the garnet-wood when uniformly sized porosity is required. 

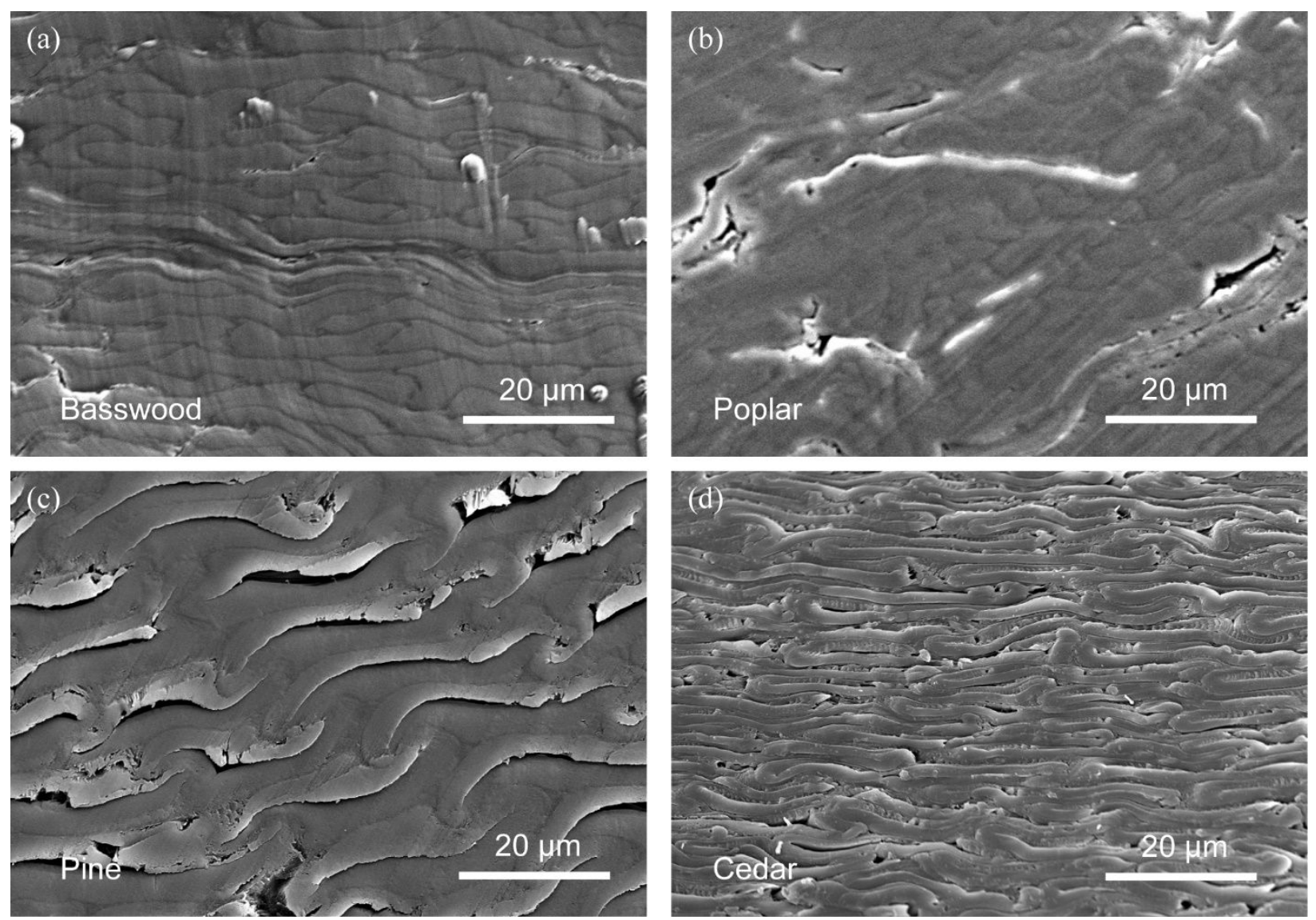

Figure S4. Cross-sectional SEM image of (a) compressed basswood, (b) compressed poplar, (c) compressed pine, and (d) compressed cedar shows similar microstructure. Though different wood species vary in microstructures, they all show a similar microstructure after compression. Regardless of the wood species, garnet membranes synthesized using wood template all have similarly aligned structure. 


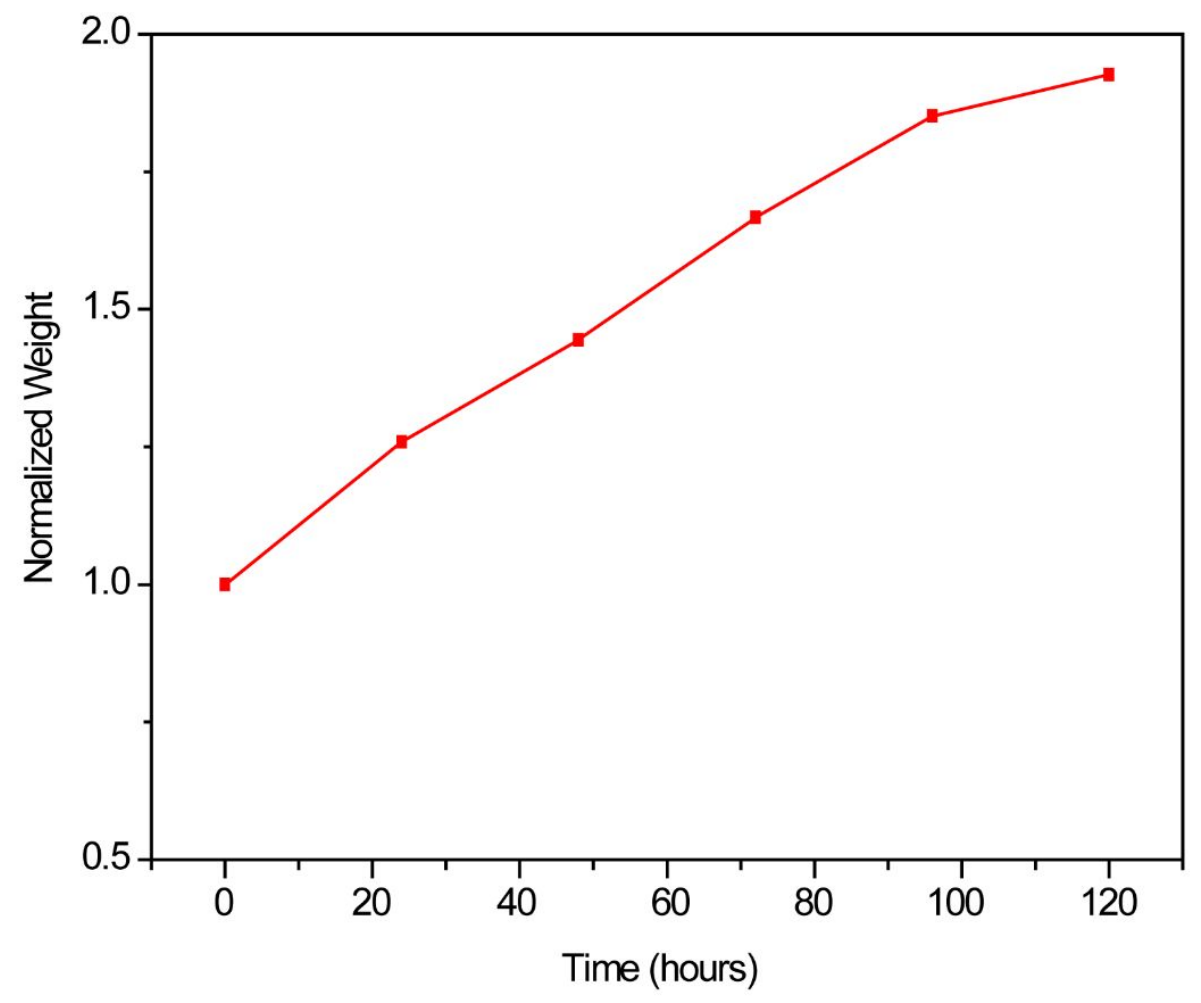

Figure S5. Weight change of the wood template during precursor infiltration. The wood template was soaked in the precursor solution and the weight increases over time. The weight change shows the high absorbency of the wood template. 


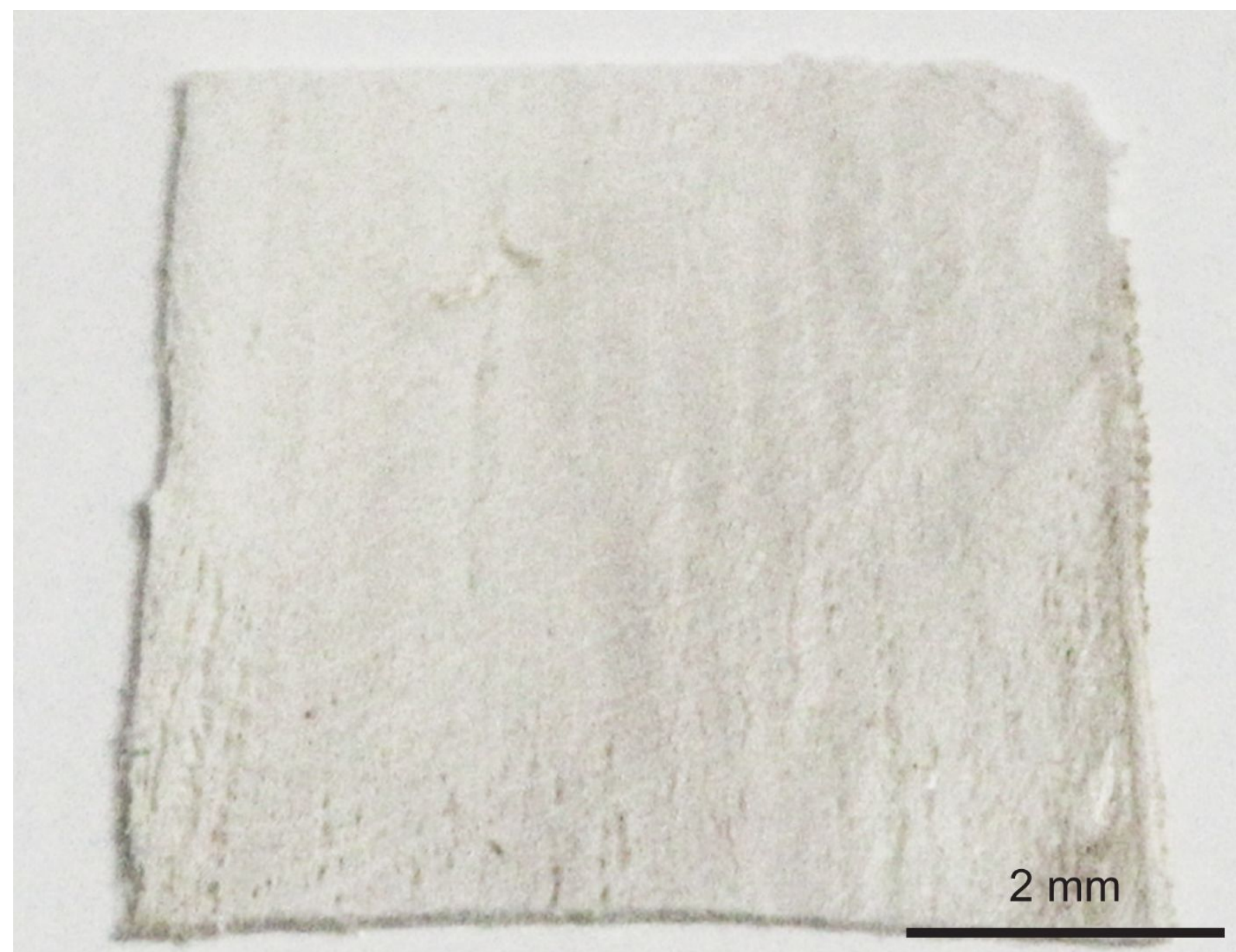

Figure S6. Photograph of an as-sintered aligned mesoporous garnet. The photograph shows a piece of the as-sintered aligned mesopores garnet. The garnet membrane was white and flat with a similar area to the wood template. 


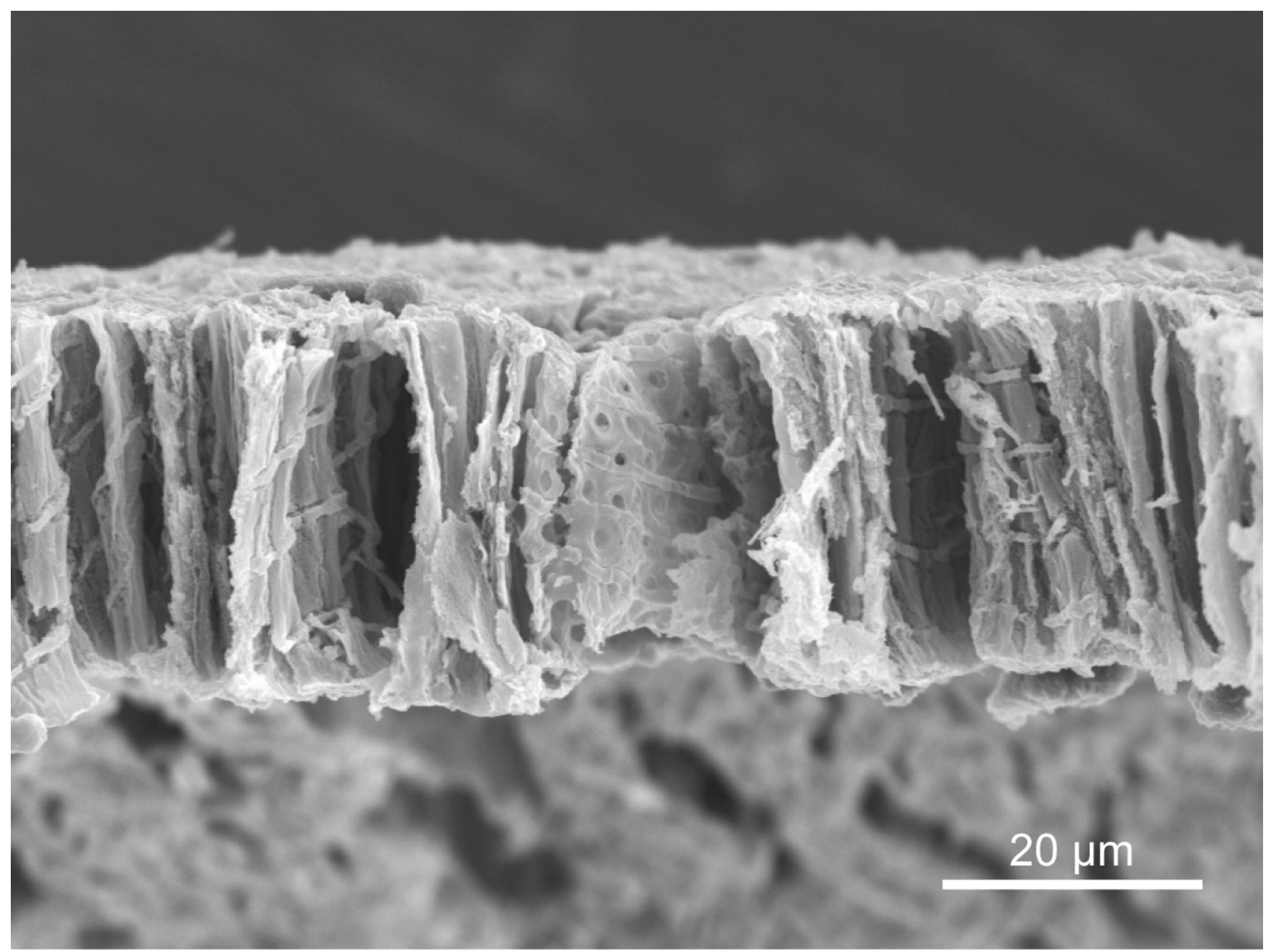

Figure S7. Cross-sectional SEM image of garnet-wood. The thickness of the garnet-wood can be controlled by the thickness of the wood template. SEM image shows a thin garnet-wood sample with a thickness of $\sim 30 \mu \mathrm{m}$, in which the low-tortuosity channels are highly aligned and penetrate throughout the whole garnet membrane. 


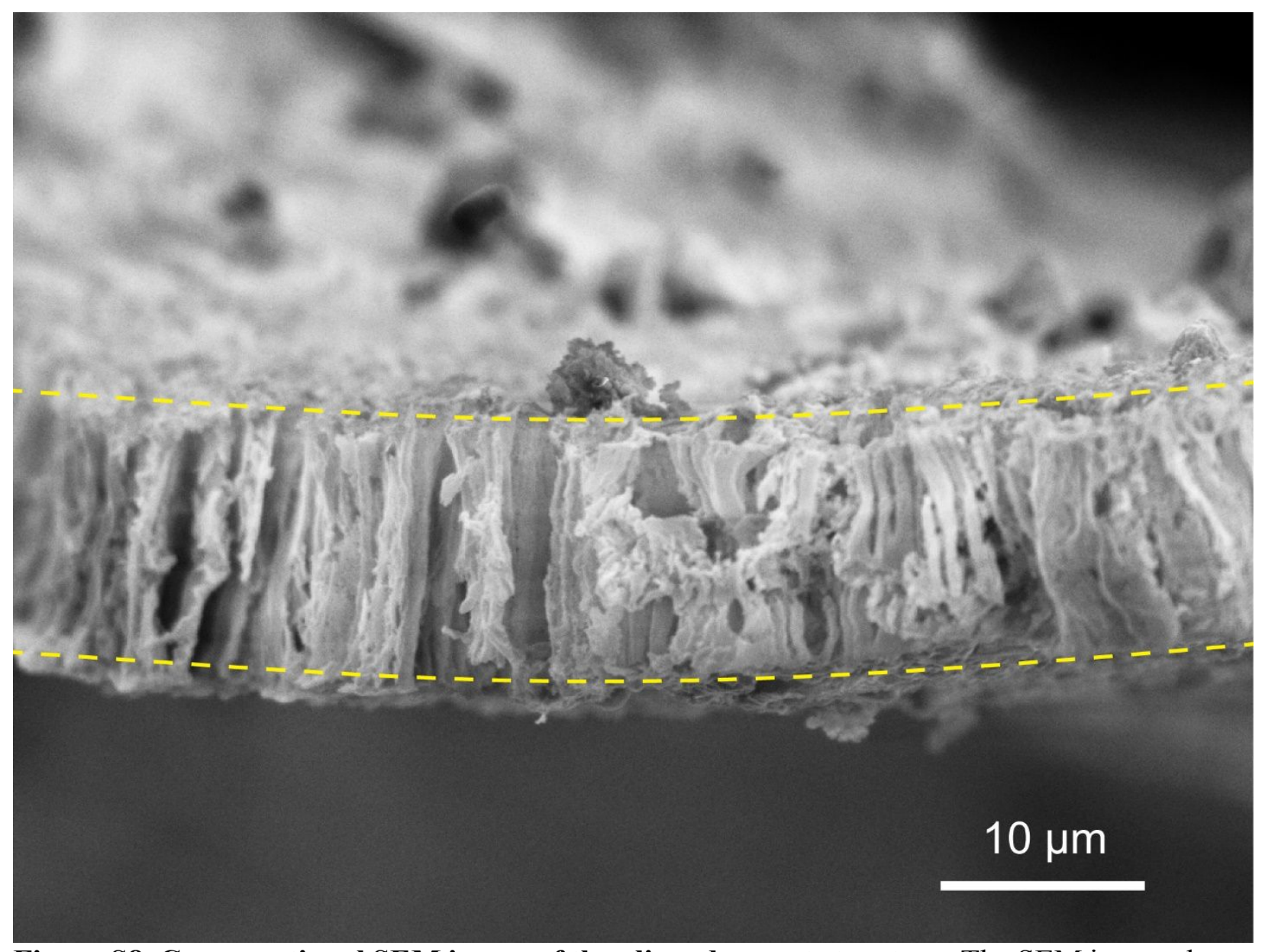

Figure S8. Cross-sectional SEM image of the aligned garnet structure. The SEM image shows a piece of garnet wood with a thickness of $18 \mu \mathrm{m}$. The sample was sintered from a template which was thinned by slicing and polishing. 


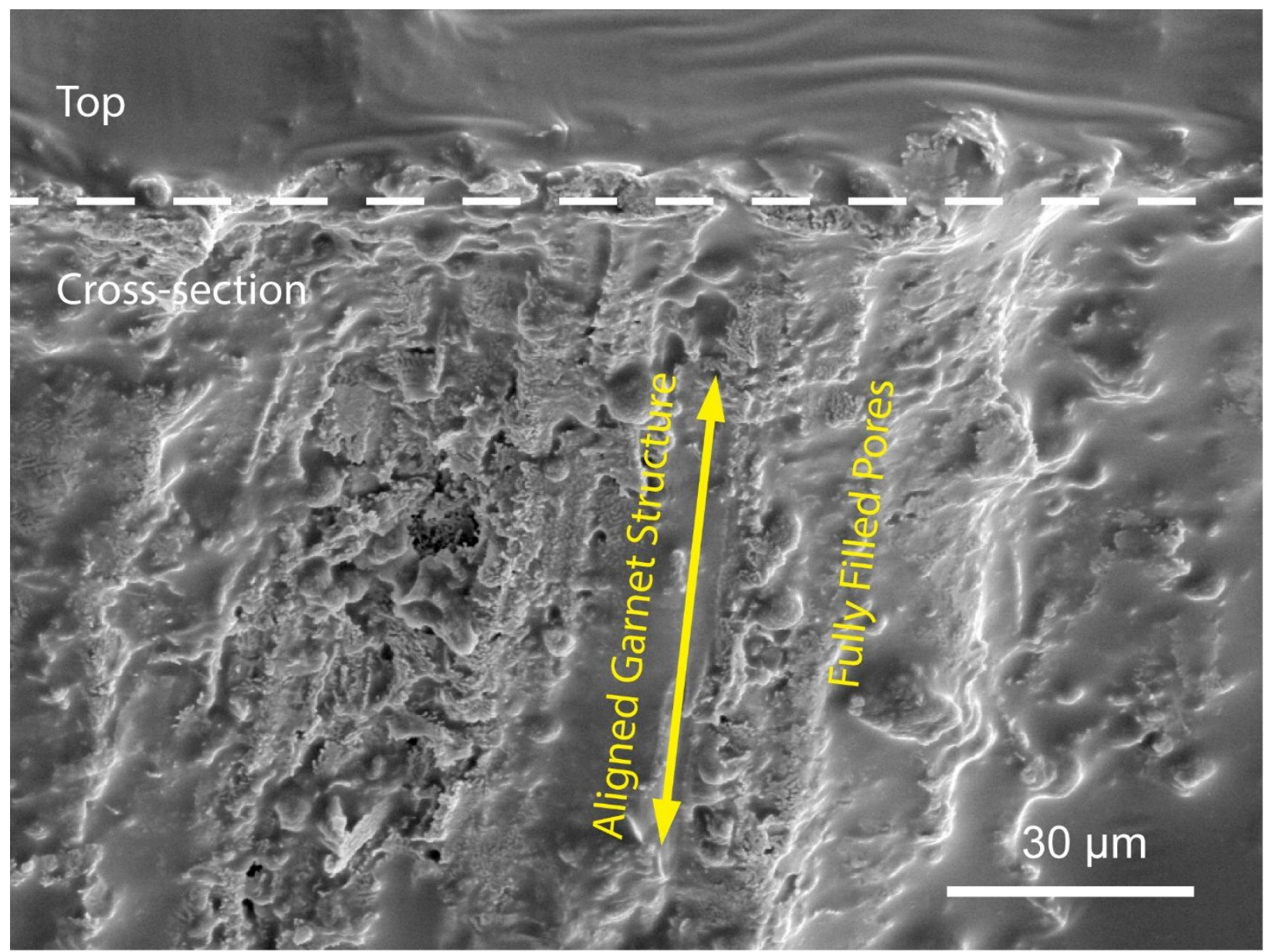

Figure S9. Cross-sectional SEM image of garnet-wood. The SEM image shows the crosssection and top surface of the garnet wood. The aligned structure is fully filled with PEO based polymer electrolyte. 

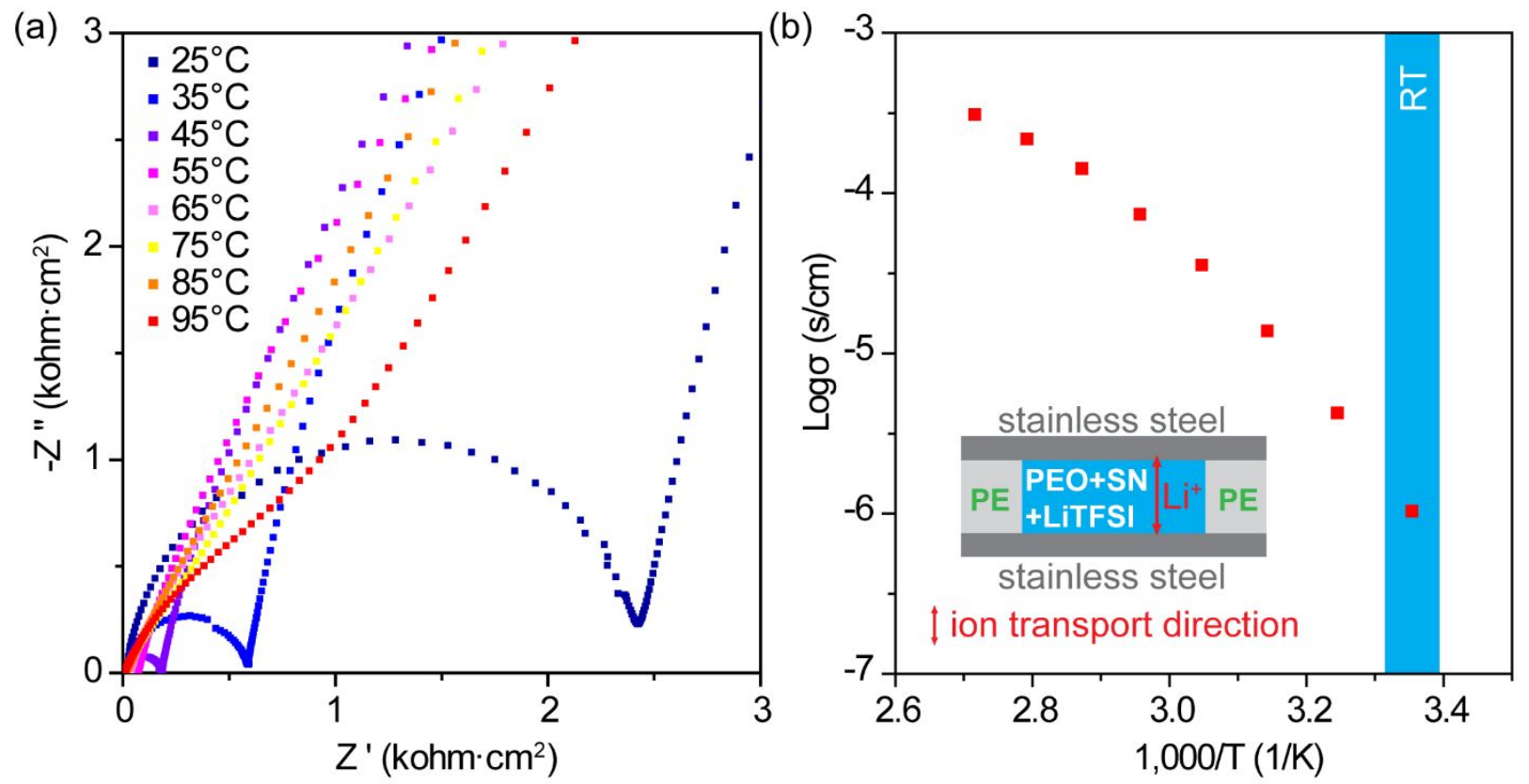

Figure S10. (a) EIS measurements and (b) ionic conductivity of the PEO/SCN/Li-TFSI polymer electrolyte at different temperatures. The blue region indicates measurements performed around room temperature (RT). The inset shows that the PEO/LiTFSI/SCN polymer electrolyte film was sandwiched by two stainless steel electrodes. A polyethylene (PE) separator ring was placed around the polymer electrolyte film to fix the thickness and avoid shorting at high temperatures. 


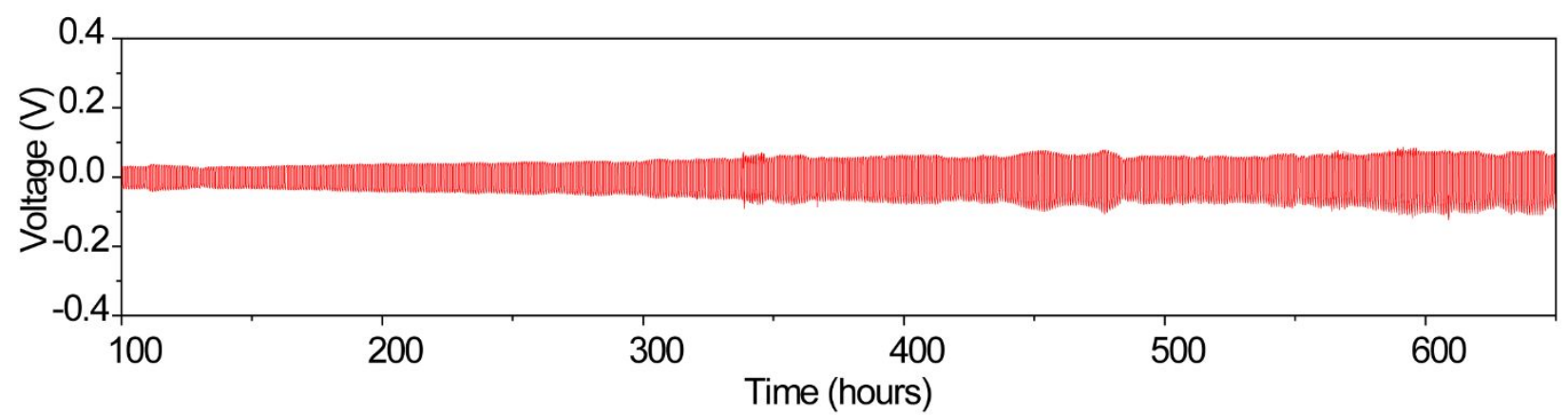

Figure S11. Galvanostatic cycling of Li/garnet $\mathrm{wood} / \mathrm{Li}$ with a current density of $0.1 \mathrm{~mA} / \mathrm{cm}^{2}$ at room temperature for over 600 hours. The sample has a thickness around $900 \mu \mathrm{m}$ and an area of about $0.1 \mathrm{~cm}^{2}$. The fluctuation in the voltage is caused by changes to the ambient temperature of the cell. The long-term cycling indicates the outstanding electrochemical and mechanical stability of the garnet wood composite electrolyte in Li metal cells. 


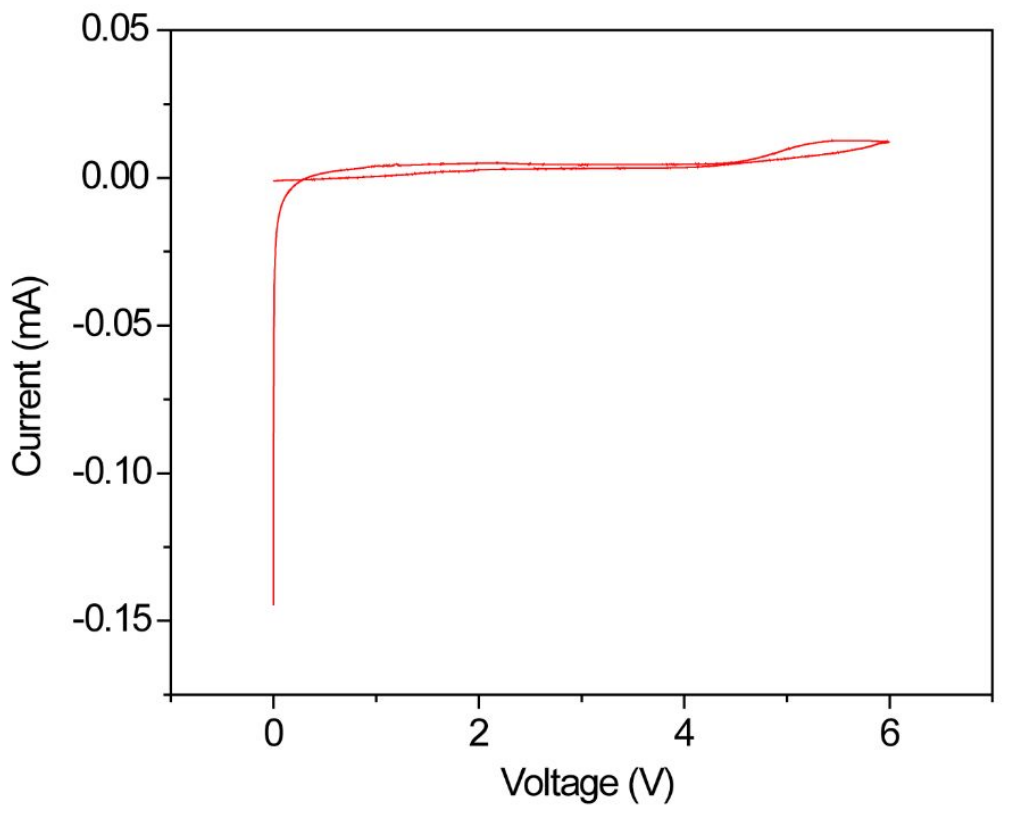

Figure S12. Cyclic voltammetry (CV) curve of garnet wood shows a wide stable voltage window up to $6 \mathrm{~V}$. Garnet wood was sandwiched with lithium metal as counter and reference electrode and stainless steel as working electrode. The wide voltage window suggests that the electrolyte meets the voltage stability requirements of high voltage oxide cathodes. 
